\title{
Solvent-free, catalyst-free Michael-type addition of amines to electron-deficient alkenes
}

\author{
Brindaban C. Ranu,* Suvendu S. Dey, and Alakananda Hajra \\ Department of Organic Chemistry, Indian Association for the Cultivation of Science, Jadavpur, \\ Calcutta - 700 032, India \\ E-mail: ocbcr@iacs.res.in
}

Dedicated to Professor S.V. Kessar on the occasion of his $70^{\text {th }}$ birthday

(received 01 May 02; accepted 30 Jun 02; published on the web $08 \mathrm{Jul} 02$ )

\begin{abstract}
A variety of amines undergo Michael-type additions to $\alpha, \beta$-unsaturated nitriles, carboxylic ester and ketones in a neat mixture without any solvent and catalyst to produce the corresponding $\beta$ amino derivatives in excellent yields.
\end{abstract}

Keywords: Michael additions, solvent-free, catalyst-free, amines, conjugated alkenes

\section{Introduction}

The conjugate addition of nucleophiles to electron-deficient alkenes is an important reaction and has wide application in organic synthesis. ${ }^{1}$ Particularly, addition of amines to $\alpha, \beta$-unsaturated carbonyl compounds and nitriles produce the $\beta$-amino derivatives which are useful intermediates in the synthesis of a large number of products with a wide range of biological activity. ${ }^{2}$ Usually, these conjugate additions are carried out in an organic solvent in the presence of a strong base ${ }^{3}$ or acid. ${ }^{4}$ To avoid disadvantages associated with such harsh catalysts a number of milder procedures using Lewis acids have been developed. ${ }^{5}$ However, use of stoichiometric amounts of Lewis acids such as $\mathrm{AlCl}_{3}, \mathrm{TiCl}_{4}$ or $\mathrm{SnCl}_{4}$ cause serious environmental problems due to strongly acidic waste streams. The development of green alternatives is therefore highly desirable and recently a couple of procedures involving clay ${ }^{6 \mathrm{a}}$ and silica gel ${ }^{6 \mathrm{~b}}$ supported conjugate addition of amines to $\alpha, \beta$-ethylenic compounds have been reported, although these reactions use solvents like dichloroethane and acetonitrile which are very high in the list of damaging chemicals. In recent times, design of solvent-free reaction has been the subject of considerable interest in the field of green synthesis ${ }^{7}$ and as a part of that goal, we like to disclose here an efficient conjugate addition of amines to electron deficient alkenes such as $\alpha, \beta$-unsaturated nitrile, carboxylic ester and ketones in neat without any solvent and catalyst. 


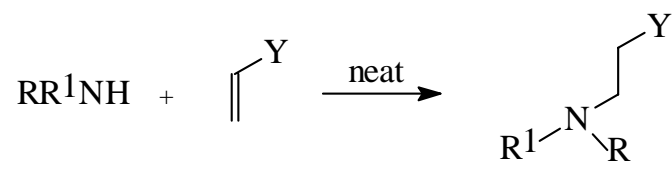

$\mathrm{Y}=\mathrm{CN}, \mathrm{CO}_{2} \mathrm{R}^{2}, \mathrm{COR}^{3}$

\section{Scheme 1}

\section{Results and Discussion}

The experimental procedure is very simple. A mixture of amine and $\alpha, \beta$-unsaturated compound was stirred at room temperature without any solvent and catalyst. The product was isolated by short column chromatography of the reaction mixture over silica gel.

Several structurally varied amines react with a variety of $\alpha, \beta$-ethylenic compounds by this procedure to produce the corresponding $\beta$-amino derivatives in excellent yields. The results are summarized in Table 1. It has been observed that cyclic secondary amines such as pyrrolidine, piperidine and morpholine undergo facile additions with acrylonitrile, acrylic esters, and cyclohexenone, while reactions of open chain secondary amines like diisopropylamine or dicyclohexylamine do not proceed at all. However, diethylamine is an exception that adds quite readily (entries 4,11). The addition of primary amines is also investigated. Aniline and other aromatic amines remained nonreactive, while aliphatic primary amines such as benzylamine and cyclohexylamine undergo efficient additions with acrylonitrile or methyl acrylate giving only the monoadduct even in the presence of excess acrylonitrile or acrylate (entries 15-17). This is not unexpected as open chain secondary amines are not reactive under these conditions. However, access to a monoadduct by this procedure is of much synthetic importance as it is difficult to get by other methods. ${ }^{6 a}$ Although the reactions of pyrrolidine and morpholine with cyclobexenone (entries 13, 14) are smooth, the addition to acylic conjugated enones like methyl vinyl ketone and mesityl oxide are rather sluggish under these conditions accomplishing conversion to the extent of $60-70 \%$ after a prolonged reaction.

In general, the reactions are reasonably fast and clean. No side product was obtained and thus no rigorous chromatographic separation or purification is required. The compounds obtained from various additions (Table 1) may be used as precursors to useful building blocks frequently used in the synthesis of important heterocyclic compounds.

In conclusion, the present solvent-free and catalyst-free Michael-type addition of amines to electron-deficient $\alpha, \beta$-unsaturated olefins provides a very simple and practical alternative to the existing procedures., ${ }^{5,6}$ Most significantly, efficiency, cost-effectiveness and green methodology will make this procedure useful to academia as well as industry. 
Table 1. Solvent - free, Catalyst - free Michael - type Addition of Amines to Electron Deficient Alkenes

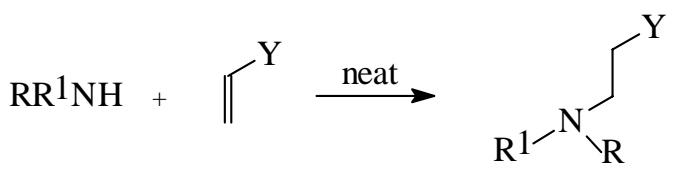

Entry




\section{Experimental Section}

General Procedures. IR spectra were taken as neat liquids. ${ }^{1} \mathrm{H}$ NMR (300 MHz) and ${ }^{13} \mathrm{C}$ NMR (75 MHz) spectra were run in $\mathrm{CDCl}_{3}$ solutions. Elemental analyses were done by a Perkin-Elmer autoanalyzer. Column chromatography was performed on silica gel (60-120 mesh, SRL, India). The amines and olefins are all commercial materials and were distilled before use.

\section{General procedure for addition of amine to conjugated alkene. Representative procedure (entry 1)}

A mixture of pyrrolidine (2.4 mmol, $170 \mathrm{mg}$ ) and acrylonitrile (2 mmol, $104 \mathrm{mg})$ was stirred at room temperature $\left(30-32^{\circ} \mathrm{C}\right)$ as a neat mixture without any solvent and catalyst for $0.75 \mathrm{~h}$ (TLC). The reaction mixture was then straightway subjected to short column chromatography over silica gel (hexane/ethyl acetate 3:1) to provide the pure 3-pyrrolidinylpropionitrile (230 mg, 93\%) as a colourless oil, IR 2964, 2798, 2246, $1483 \mathrm{~cm}^{-1}$; ${ }^{1} \mathrm{H}$ NMR $\delta 2.67$ (t, $\left.J=7.0 \mathrm{~Hz}, 2 \mathrm{H}\right), 2.45$ (m, 6H), 1.69 (m, 4H); ${ }^{13} \mathrm{C}$ NMR $\delta$ 119.2, 54.1 (2C), 51.5, 23.8 (2C), 17.9. Anal. Calcd for $\mathrm{C}_{7} \mathrm{H}_{12} \mathrm{~N}_{2}: \mathrm{C}$, 67.70; H, 9.74; N, 22.56. Found : C, 67.53; H, 9.68; N, 22.35.

This procedure is followed for all reactions listed in Table 1.

Spectral and analytical data of the new compounds, designated by their entries in Table 1.

3-Phenyl-3-pyrrolidinyl-propionitrile (entry 2) . Colourless oil; IR (neat) 2968, $2246 \mathrm{~cm}^{-1}$; ${ }^{1} \mathrm{HNMR} \delta$ 7.42-7.28 (m, 5H), 3.47 (t, $\left.J=6.0 \mathrm{~Hz}, 1 \mathrm{H}\right), 2.83-2.77$ (m, 2H), 2.59-2.45 (m, 4H), 1.80 (m, 4H). Anal. Calcd for $\mathrm{C}_{13} \mathrm{H}_{16} \mathrm{~N}_{2}$ : C, 77.96; H, 8.05; N, 13.99. Found : C, 77.84; H, 8.03; $\mathrm{N}, 13.77$.

3-( $N, N$-Diethylamino)-propionitrile (entry 4). Colourless oil; IR (neat) 2975, $2246 \mathrm{~cm}^{-1} ;{ }^{1} \mathrm{H}$ NMR $\delta 2.65(\mathrm{t}, J=7.0 \mathrm{~Hz}, 2 \mathrm{H}), 2.41$ (q, $J=7.1 \mathrm{~Hz}, 4 \mathrm{H}), 2.31$ (t, $J=7.0 \mathrm{~Hz}, 2 \mathrm{H}), 0.91$ (t, $J=7.1$ $\mathrm{Hz}, 6 \mathrm{H}) ;{ }^{13} \mathrm{C}$ NMR $\delta$ 119.4, 48.6, 47.0 (2C), 16.5, 12.2 (2C). Anal. Calcd for $\mathrm{C}_{7} \mathrm{H}_{14} \mathrm{~N}_{2}: \mathrm{C}, 66.62$; H, 11.18; N, 22.20. Found : C, 66.50; H, 11.08; N, 22.12.

Methyl 3-pyrrolidinyl-propionate (entry 5) . Colourless oil; IR (neat) 2954, $1739 \mathrm{~cm}^{-1} ;{ }^{1} \mathrm{H}$ NMR $\delta 3.67(\mathrm{~s}, 3 \mathrm{H}), 2.86(\mathrm{t}, J=7.0 \mathrm{~Hz}, 2 \mathrm{H}), 2.34(\mathrm{~m}, 6 \mathrm{H}), 1.17(\mathrm{~m}, 4 \mathrm{H}) ;{ }^{13} \mathrm{C}$ NMR $\delta 172.9$, 54.1 (2C), 51.6, 51.5, 34.1, 23.6 (2C). Anal.Calcd for $\mathrm{C}_{8} \mathrm{H}_{15} \mathrm{NO}_{2}$ : C, 61.12; H, 9.62; N, 8.91. Found : C, 61.25; H, 9.41; N, 8.70.

Methyl (2-methyl-3-prrrolidinyl)-propionate (entry 6) . Colourless oil; IR (neat) 2968, $1739 \mathrm{~cm}^{-1}$; ${ }^{1} \mathrm{H}$ NMR $\delta 3.68$ (s, 3H), 2.83-2.42 (m, 7H), $1.76(\mathrm{~m}, 4 \mathrm{H}), 1.16$ (d, $\left.J=8.3 \mathrm{~Hz}, 3 \mathrm{H}\right)$; ${ }^{13}$ c NMR $\delta$ 176.8, 59.8, 54.5 (2C), 51.8, 39.8, 23.8 (2C), 16.1. Anal. Calcd for $\mathrm{C}_{9} \mathrm{H}_{17} \mathrm{NO}_{2}: \mathrm{C}$, 63.13; H, 10.01; N, 8.18. Found : C, 63.32; H, 10.14; N, 8.28.

Ethyl 3-(3-methoxyphenyl)-3-pyrrolidinyl-propionate (entry 7). Colourless oil; IR (neat) 2968, $1735 \mathrm{~cm}^{-1}$; ${ }^{1} \mathrm{H}$ NMR $\delta 7.20$ (t, $\left.J=8.0 \mathrm{~Hz}, 1 \mathrm{H}\right), 6.93-6.90$ (m, 2H), 6.80-6.76 (m, 1H), 3.98 (q, $J=7.1 \mathrm{~Hz}, 2 \mathrm{H}), 3.79$ (s, 3H), 3.71-3.66 (m, 1H), 2.96 (dd, $J=14.61,5.76 \mathrm{~Hz}, 1 \mathrm{H}$ ), 2.69 (dd, $J=14.64,8.76 \mathrm{~Hz}, 1 \mathrm{H}), 2.72-2.40(\mathrm{~m}, 4 \mathrm{H}), 1.75(\mathrm{~m}, 4 \mathrm{H}), 1.09(\mathrm{t}, J=7.1 \mathrm{~Hz}, 3 \mathrm{H}) ;{ }^{13} \mathrm{C}$ NMR $\delta$ 171.8, 159.9, 143.6, 129.4, 120.8, 113.8, 113.3, 66.8, 60.6, 55.5, 52.5 (2C), 42.0, 23.7 (2C), 14.4. Anal. Calcd for $\mathrm{C}_{16} \mathrm{H}_{23} \mathrm{NO}_{3}$ : C, 69.29; H, 8.36; N, 5.05. Found : C, 69.48; H, 8.26; N, 5.14. 
Ethyl 3-methyl-3-pyrrolidinyl-propionate (entry 8). Colourless oil; IR (neat) 2968, $1739 \mathrm{~cm}^{-1}$; ${ }^{1} \mathrm{H}$ NMR $\delta 4.12(\mathrm{q}, J=7.1 \mathrm{~Hz}, 2 \mathrm{H}), 2.90(\mathrm{~m}, 1 \mathrm{H}), 2.70-2.61(\mathrm{~m}, 5 \mathrm{H}), 2.33-$ $2.24(\mathrm{~m}, 1 \mathrm{H}), 1.77(\mathrm{~m}, 4 \mathrm{H}), 1.24$ (t, $J=7.1 \mathrm{~Hz}, 3 \mathrm{H}), 1.15$ (d, $J=6.3 \mathrm{~Hz}, 3 \mathrm{H})$. Anal. Calcd for $\mathrm{C}_{10} \mathrm{H}_{19} \mathrm{NO}_{2}$ : C, 64.83; H, 10.34; N, 7.56. Found : c, 64.64; H, 10.38; N, 7.75.

Methyl 3-piperidinyl-propionate (entry 9). Colourless oil; IR (neat) 2935, $1741 \mathrm{~cm}^{-1}$; ${ }^{1} \mathrm{H}$ NMR $\delta 3.62$ (s, 3H), $2.61(\mathrm{t}, J=8.0 \mathrm{~Hz}, 2 \mathrm{H}), 2.47$ (t, $J=7.9 \mathrm{~Hz}, 2 \mathrm{H}), 2.34(\mathrm{~m}, 4 \mathrm{H}), 1.55-1.36$ $(\mathrm{m}, 6 \mathrm{H}) ;{ }^{13} \mathrm{C}$ NMR $\delta$ 173.5, 54.6, 54.5 (2C), 51.9, 32.3, 26.2 (2C), 24.5. Anal. Calcd for $\mathrm{C}_{9} \mathrm{H}_{17} \mathrm{NO}_{2}$ : C, 63.13; H, 10.01; N, 8.18. Found : c, 63.43; H, 10.08; N, 8.36.

Methyl 3-(3-methoxyphenyl)-3-piperidinyl-propionate (entry 10). Colourless oil; IR (neat) 2935, $1739 \mathrm{~cm}^{-1}$; ${ }^{1} \mathrm{H}$ NMR $\delta$ 7.26-7.20 (m, 1H); 6.84-6.80 (m, 3H), 3.97 (t, $\left.J=7.5 \mathrm{~Hz}, 1 \mathrm{H}\right), 3.81$ (s, 3H), 3.61 (s, 3H), 2.99 (dd, $J=14.8,7.4 \mathrm{~Hz}, 1 \mathrm{H}$ ), 2.70 (dd, $J=14.8,7.7 \mathrm{~Hz}, 1 \mathrm{H}$ ), 2.46-2.32 (m, 4H), 1.55-1.26 (m, 6H). Anal. Calcd for $\mathrm{C}_{16} \mathrm{H}_{23} \mathrm{NO}_{3}$ : C, 69.29; H, 8.36; N, 5.05. Found : C, 69.07; H, 8.04; N, 5.24.

Methyl (3- $N, N$-diethylamino)propionate (entry 11). Colourless oil; IR (neat) $2970,1741 \mathrm{~cm}^{-1} ;{ }^{1} \mathrm{H}$ NMR $\delta 3.57$ (s, 3H), 2.70 (t, $J=7.1 \mathrm{~Hz}, 2 \mathrm{H}), 2.46-2.34$ (m, 6H), 0.93 (t, $J=7.1 \mathrm{~Hz}, 6 \mathrm{H}) ;{ }^{13} \mathrm{C}$ NMR $\delta 173.4,51.7$, 48.3, 47.11 (2C), 32.4, 12.0 (2C). Anal. Calcd for $\mathrm{C}_{8} \mathrm{H}_{17} \mathrm{NO}_{2}$ : C, 60.35; H, 10.76; N, 8.80. Found : C, 60.24; H, 10.64; N, 8.62.

Methyl 2-methyl-3-morpholin-4-yl-propionate (entry 12). Colourless oil; IR (neat) 2952, $1739 \mathrm{~cm}^{-1}$; ${ }^{1} \mathrm{H}$ NMR $\delta 3.59$ (s, 3H), 3.58-3.56 (m, 4H), 2.64-2.19 (m, 7H), 1.07 (d, $J=6.6 \mathrm{~Hz}, 3 \mathrm{H}) ;{ }^{13} \mathrm{C}$ NMR $\delta$ 176.6, 67.2 (2C), 62.3, 54.0 (2C), 51.8, 37.8, 15.8. Anal. Calcd for $\mathrm{C}_{9} \mathrm{H}_{17} \mathrm{NO}_{3}$ : C, 57.73; H, 9.15; N, 7.48. Found : C, 57.52; H, 9.04; N, 7.36.

3-Pyrrolidinylcyclohexanone (entry 13). Colourless oil; IR (neat) 2950,1708 cm ${ }^{-1}$; ${ }^{1} \mathrm{H}$ NM $\delta$ 2.59-2.19 (m, 9H), 2.01-1.92 (m, 2H), 1.79-1.53 (m, 6H); ${ }^{13} \mathrm{C}$ NMR $\delta$ 210.7, 63.7, 54.2 (2C), 44.7, 41.5, 28.1, 23.9 (2C), 22.6. Anal. Calcd for $\mathrm{C}_{10} \mathrm{H}_{17} \mathrm{NO}$ : C, 71.81; H, 10.25; N, 8.37. Found : C, 71.62; H, 10.04; N, 8.25.

3-Morpholine-4-yl-cyclohexanone (entry 14). Colourless oil; IR (neat) 2950, $1708 \mathrm{~cm}^{-1} ;{ }^{1} \mathrm{H}$ NMR $\delta 3.63$ (m, 4H), 2.59-2.19 (m, 9H), 2.01-1.92 (m, 2H), 1.66-1.53 (m, 2H); ${ }^{13} \mathrm{C}$ NMR $\delta 210.7,67.4$ (2C), 63.7, 49.9 (2C), 44.6, 41.5, 28.1, 22.6. Anal. Calcd for $\mathrm{C}_{10} \mathrm{H}_{17} \mathrm{NO}_{2}$ : C, 65.54; H, 9.35; N, 7.64. Found : C, 65.23; H, 9.39; N, 7.42 .

Methyl (3-cyclohexylamino)-propionate (entry 17). Colourless oil; IR (neat) 3311, $1739 \mathrm{~cm}^{-1}$; ${ }^{1} \mathrm{H}$ NMR $\delta 3.60$ (s, 3H), 2.83 (t, $J=6.6 \mathrm{~Hz}, 2 \mathrm{H}), 2.43$ (t, $J=6.6 \mathrm{~Hz}, 2 \mathrm{H}), 2.37-2.31$ (m, 1H), 1.79 (broad s, 1H), 1.671.52 (m, 5H), 1.24-0.93 (m, 5H); ${ }^{13} \mathrm{C}$ NMR $\delta$ 173.5, 56.8, 51.8, 42.3, 35.1, 33.7 (2C), 26.4, 25.3 (2C). Anal. Calcd for $\mathrm{C}_{10} \mathrm{H}_{19} \mathrm{NO}_{2}$ : C, 64.83; H, 10.34; N, 7.56. Found : C, 64.62; H, 10.23; N, 7.35.

\section{Acknowledgements}

This investigation has enjoyed financial support from CSIR (Grant No. 01(1739)/02). SSD and $\mathrm{AH}$ are also thankful to CSIR for their fellowships. 


\section{References}

1. Perlmutter, P. Conjugated Addition Reactions in Organic Synthesis; Pergamon Press: Oxford, 1992, p 114.

2. (a) Nicolaou, K. C.; Dai, V.-M.; Guy, R. K. Angew. Chem. Int. Ed. 1994, 33, 15. (b) Frackenpohl, J.; Arridson, P. I.; Screiber, J. V.; Seebach, D., Chem. Bio.Chem. 2001, 2, 445.

3. Davies, S.G.; McCarthy, T.D. Synlett 1995, 700.

4. Rosenthal, D.; Braundrup, G.; Davies, K. H.; Wall, M. E. J. Org. Chem. 1965, 30, 3689.

5. (a) Ben Ayed, T.; Amiri, H.; El Gaied, M. M.; Villieras, J. Tetrahedron 1995, 35, 9633 and references cited therein. (b) Loh,T. P.; Wei, L.-L. Synlett 1998, 975.

6. (a) Shaikh, N. S.; Deshpande, V.H.; Bedekar, A. V. Tetrahedron 2001, 57, 9045. (b) Bartoli, G.; Bosco, M.; Marcantoni, E.; Petrini, M.; Sambri, L.; Torregiani, E. J. Org. Chem. 2001, 66, 9052.

7. (a) Tanaka, K.; Toda, F. Chem. Rev. 2000, 100, 1025. (b) Cave, G. W. V.; Raston, C. L.; Scott. J. L. Chem. Commun. 2001, 2159. (c) Metzger, J. O. Angew. Chem., Int. Ed. 1998, 37, 2975. 